\title{
Preparation and characterization of cationic Pluronic for surface modification and functionalization of polymeric drug delivery nanoparticles
}

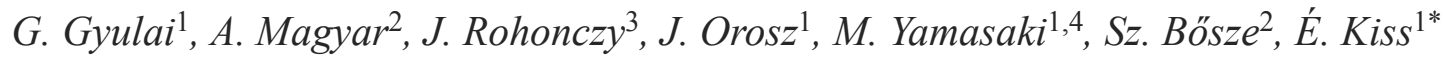 \\ ${ }^{1}$ Laboratory of Interfaces and Nanostructures, Institute of Chemistry, Eötvös Loránd University, Budapest 112, \\ PO Box 32, H-1518 Budapest, Hungary \\ ${ }^{2}$ MTA-ELTE Research Group of Peptide Chemistry, Budapest 112, PO Box 32, H-1518 Budapest, Hungary \\ ${ }^{3}$ Department of Inorganic Chemistry, Institute of Chemistry, Eötvös Loránd University, Budapest 112, PO Box 32, \\ H-1518 Budapest, Hungary \\ ${ }^{4}$ GENMAT - Chemistry Department, Federal University of Mato Grosso, Cuiabá/MT - 78060-900, MT - Brazil
}

Received 16 July 2015; accepted in revised form 4 October 2015

\begin{abstract}
Biodegradable poly(lactic-co-glycolic acid) copolymer, PLGA nanoparticles (NPs) with a surface layer of poly (ethylene oxide)-poly(propylene oxide)-poly(ethylene oxide) triblock copolymers, Pluronics, are promising drug carrier systems. With the aim to increase the potential of targeted drug delivery the end group derivative of Pluronics was synthesized in a straightforward way to obtain Pluronic-amines. The formation of functional amine groups was confirmed by fluorescamine method and NMR analysis of their N-(tert-Butoxycarbonyl)-L-phenylalanine (Boc-Phe-OH) and N-(9-Fluorenylmethoxycarbonyl)-L-phenylalanine (Fmoc-Phe-OH) conjugates. Pluronic and Pluronic-amine stabilized PLGA NPs prepared by nanoprecipitation were characterized by dynamic light scattering and zeta potential measurements. All of the systems showed high colloidal stability checked by electrolyte induced aggregation, although the presence of Pluronicamine on the surface decreased the zeta potential in some extent. The introduction of reactive primary amine groups into the surface layer of PLGA NPs while preserving the aggregation stability, provides a possibility for coupling of various ligands allowing targeted delivery and also contributes to the improved membrane affinity of NPs.
\end{abstract}

Keywords: biocompatible polymers, Pluronic derivatization, PLGA nanoparticles, colloidal stability, polymeric drug delivery

\section{Introduction}

Extensive research went into the development of drug delivery systems in the past decades to reduce the side effects and enhance the therapeutic efficacy of drugs [1]. Biodegradable polymeric nanoparticles (NPs) offer the possibility for prolonged drug release as well as targeted delivery. Poly(lactic-co-glycolic acid) copolymers (PLGA) are preferred biomaterials because of their nontoxicity, biocompatibility and biodegradability [2-6]. PLGA readily forms NPs via the nanoprecipitation method as described by Fessi et al. [7]. In most cases the drug is encapsulated into the polymeric particle and released in a controlled way by diffusion and erosion of the polymeric matrix [8-11]. It was found however, that the hydrophobic character of the PLGA triggers the nonspecific adsorption of plasma proteins leading to the uptake of the particles by the mononuclear phagocyte system and hence their fast clearance from the body [12-14]. This undesirable process can be prevented by forming a poly(ethylene oxide), PEO corona on the particle to improve its surface biocompatibility [15-18]. Among the various techniques developed for surface immobilization of PEO the

\footnotetext{
${ }^{*}$ Corresponding author, e-mail: kisseva@chem.elte.hu

(C) BME-PT
} 
adsorption of poly(ethylene oxide)-poly(propylene oxide)-poly(ethylene oxide) triblock copolymers, the use of Pluronics is a versatile and convenient method to obtain PEO-rich surface on PLGA NPs. The hydrophobic PPO part of the molecule facilitates the anchorage of the surface modifier to the PLGA while the PEO chains form an outer layer introducing steric stability to the NPs [19-22]. In addition to reduced protein adsorption and reasonable colloidal stability the capability of NPs to cross cell membranes and intracellular barriers is also a requirement in drug carrier applications. Previous studies demonstrated that the presence of certain Pluronics on the surface of the PLGA NPs enhances the nonspecific membrane affinity of the system [23]. The specific targeting needs the conjugation of appropriate biospecies to the surface of NPs. PLGA particles contain chain end carboxylic groups on their surface but these are small in number and not easily accessible because of the presence of the Pluronic molecules on the surface. The surface Pluronic layer on the other hand contains only hydroxyl groups at the chain ends which have limited reactivity under normal conditions. Introduction of reactive amino groups onto the surface would provide a convenient way for immobilization of the desired ligand.

Transformation of Pluronic end groups into amine is the aim of our work and furthermore to reveal whether this change influences the physico-chemical and drug carrier properties of the Pluronic stabilized PLGA NPs.

Formation of amine end groups on PEO molecules can be achieved by various methods which usually involve two or more reaction steps. One of those is the conversion of the hydroxyl groups to halide or sulfonyl ester, followed by a reaction with excess amount of ammonia [24, 25]. Another strategy for the introduction of amine functionality is by activating the hydroxyl groups followed by conjugation with diamines and polyamines [26, 27]. A problem with these reactions is the possibility for secondary amine side product formation as well as the risk of crosslinking [28]. Harris et al. [29] described a straightforward, two step reaction for the preparation of PEOamines that involved mild reaction conditions with relatively safe reactants. Hydroxyl group is oxydised to aldehyde followed by reaction with ammonia in the presence of sodium cyanoborohydride.

We adapted the latest procedure to the preparation of amine derivative of Pluronic block copolymers. The advantage of these cationic derivatives in surface modification can be twofold. The introduction of primary amine groups provides the possibility of further coupling allowing e.g. the surface immobilization of targeting ligands on the NP surface. In addition to that the presence of positively charged molecules on a nanoparticle surface enhances their nonspecific membrane affinity $[23,30]$. It is however, an open question how these cationic derivatives change the physico-chemical properties of PLGA NPs compared to the systems stabilized with Pluronics. The synthesis of Pluronic-amines and their application in the preparation of surface modified PLGA NPs relevant as drug carriers is described in the present work. The nanoparticles were characterized by their size, surface charge and colloidal stability.

\section{Experimental}

\subsection{Materials}

Poly(ethylene oxide)/poly(propylene oxide)/poly (ethyleneoxide), PEO-PPO-PEO triblock copolymers, Pluronic F68 (M:8400), Pluronic F127 (M:12600) and Pluronic F108 (M:14600) (provided by BASF Hungaria Kft.), were applied as received. The composition of the polymers is characterized by the average length of the PPO chain $(27,56$ and 44 monomeric units for F68, F127, and F108, respectively) and the length of the PEO chains (80, 101, and 141 monomeric units for F68, F127, and F108, respectively).

Poly(D,L-lactic-co-glycolic acid), PLGA with $50 \%$ of lactic and $50 \%$ of glycolic acid content (M:5000075000) was obtained from Sigma-Aldrich. Acetic anhydride, ammonium chloride, potassium hydroxide and sodium cyanoborohydride used for the chemical modification of the Pluronics were purchased from Reanal Ltd. (Budapest, Hungary). Fluorescamine, hexylamine and pyrene used in the characterization of Pluronics were acquired from Sigma-Aldrich.

N-(tert-butoxycarbonyl)-L-phenylalanine,9-fluorenylmethoxycarbonyl-L-phenylalanine, isobutyl-chloroformate(Sigma-Aldrich Kft, Budapest, Hungary) and N-methylmorpholine (Fluka,Buchs, Switzerland) were used for conjugation. Sodium chloride (Riedel de Haën), calcium acetate (Reanal Ltd.) and all solvents were of analytical grade.

\subsection{Preparation of Pluronic-amines}

Primary amine terminated Pluronic derivatives were synthesized according to the method described by 
Harris et al. [29] for the modification of polyethylene oxides. First $0.4 \mathrm{mmol}$ Pluronic was dissolved in $15 \mathrm{~mL}$ freshly distilled DMSO. Following complete dissolution $3.7 \mathrm{mmol}$ acetic anhydride was added and the reaction mixture was stirred for $30 \mathrm{~h}$ at room temperature. During this step the hydroxyl end groups of the Pluronic were oxidized into aldehyde form [31]. The reaction was stopped by precipitating the polymer in cold diethyl ether. After filtration the polymer was re-dissolved in dichloromethane then precipitated again in ether. The appearance of aldehyde groups was confirmed using Schiff reagent [32]. Pluronic sample was added to the reagent and the development of a rose color indicated the presence of aldehyde groups.

0.15 mmol Pluronic-aldehyde with $3 \mathrm{mmol}$ ammonium-chloride, and $0.02 \mathrm{~g}$ potassium hydroxide was dissolved in $5 \mathrm{~mL}$ methanol. $3 \mathrm{mmol}$ sodium cyanoborohydride in $5 \mathrm{~mL}$ methanol was added to this solution slowly over $30 \mathrm{~min}$. Then $0.1 \mathrm{~g}$ potassium hydroxide was added to the reaction mixture which was stirred for $24 \mathrm{~h}$ at room temperature. The reaction was stopped by precipitating the product in cold ether. After filtration the polymer was dissolved in water and purified by dialysis against double distilled water. The purified product was acquired following the liophilization of the aqueous solution. The Pluronic-amines were prepared in three independent batches.

As a comparison the reactions were also carried out using water as a solvent instead of methanol.

\subsubsection{Fluorescamine assay}

The transformation of hydroxyl groups of Pluronic into amine groups was detected with the aid of the fluorescamine assay, which is a sensitive method for the surveying of primary amines [33-35]. Samples were prepared in $0.1 \mathrm{M}$ borate buffer at $\mathrm{pH} 9$ with a polymer concentration of $0.2 \mathrm{~g} / \mathrm{L} .150 \mu \mathrm{L}$ fluorescamine solution in acetone with a concentration of $0.3 \mathrm{~g} / \mathrm{L}$ was added to $3 \mathrm{~mL}$ of sample solution. The solution was vortexed for $10 \mathrm{~s}$ at $1000 \mathrm{rpm}$. Fluorescent spectra of the samples were recorded with a Varian Cary Eclipse fluorescence spectrophotometer (right-angle geometry, $1 \times 1 \mathrm{~cm}^{2}$ quartz cell). Monochromator slits were set to $5 \mathrm{~nm}$ and the excitation wavelength was $390 \mathrm{~nm}$. Emission of the fluorescamine derivative of the Pluronic-amine was recorded at $486 \mathrm{~nm}$. Degree of conversion of the end groups to amine was estimated from measurements using hexylamine as reference material. The measurements were carried out in triplicates.

\subsubsection{NMR assay}

All NMR spectra were obtained using a BRUKER AVANCE III 500 spectrometer operating at $500131 \mathrm{MHz}$ for the observation of ${ }^{1} \mathrm{H}$ nuclei in a $5 \mathrm{~mm}$ inverse probe. All spectra were recorded at $27^{\circ} \mathrm{C}$ in deuterated chloroform as the lock solvent. To quantify the spectra a long repetition time of $8.3 \mathrm{~s}$ and $30^{\circ}$ pulse angle were used. 4096 transients were accumulated in order to get excellent sensitivity.

\subsubsection{Conjugation with amino acid}

The N-(tert-butoxycarbonyl)-L-phenylalanine (BocPhe-OH) and 9-fluorenylmethoxycarbonyl-L-phenylalanine (Fmoc-Phe-OH) analogue of Pluronic F127amine were synthesized manually using mixed anhydride method. The polymer was dissolved in ice cold dimethylformamide ( $20 \mathrm{wt} \%)$ and 500 equiv of the N-terminus protected amino acid and 550 equiv isobutyl-chloroformate and 4-methylmorpholinein dimethylformamide were added. The mixture was stirred for $2 \mathrm{~h}$ at room temperature then left in refrigerator overnight at $4^{\circ} \mathrm{C}$. The resulting polymer-derivatives were purified by repeated dialysis against doubly distilled water (Slide-A-Lyzer G2 Dialysis Casette, MWCO: 10 kDa, ThermoScientic, USA).

\subsubsection{Determination of critical micelle concentration}

The critical micelle concentration (CMC) of Pluronics and Pluronic-amines was determined by fluorimetric method using pyrene as fluorescence probe. Pyrene is a hydrophobic fluorescence dye which shows different spectral pattern in micellar and nonmicellar solution due its sensitivity to the polarity of the medium. $500 \mu \mathrm{L}$ of pyrene solution in acetone with a concentration of $1.2 \mathrm{mM}$ was added to $1 \mathrm{~L}$ of doubly distilled water and left overnight under stirring. A series of Pluronic solutions with increasing concentration were prepared using the filtered pyrene solution as medium. The concentration range was selected according to the CMC of the various Pluronics reported previously (Table 1) [36]. Samples were allowed to equilibrate for $1 \mathrm{~h}$. The measurements were carried out with a Varian Cary Eclipse fluorescence spectrophotometer (right-angle geometry, $1 \times 1 \mathrm{~cm}^{2}$ quartz cell) at 25 and $37^{\circ} \mathrm{C}$. The exci- 
Table 1. Critical micelle concentration of Pluronics and their amine derivatives

\begin{tabular}{|l|c|c|c|}
\hline \multicolumn{1}{|c|}{ Sample } & $\begin{array}{c}\mathbf{C M C}^{*} \\
\left(\mathbf{2 5}^{\circ} \mathbf{C}\right) \\
{[\mathbf{g} / \mathbf{L}]}\end{array}$ & $\begin{array}{c}\mathbf{C M C} \\
\left(\mathbf{2 5}^{\circ} \mathbf{C}\right) \\
{[\mathbf{g} / \mathbf{L}]}\end{array}$ & $\begin{array}{c}\mathbf{C M C} \\
\left(\mathbf{3 7}^{\circ} \mathbf{C}\right) \\
{[\mathbf{g} / \mathbf{L}]}\end{array}$ \\
\hline Pluronic F68 & 190 & $>100$ & $>100$ \\
\hline Pluronic F68-amine & - & $>100$ & $>100$ \\
\hline Pluronic F127 & $1-7$ & 3.3 & 0.09 \\
\hline Pluronic F127-amine & - & 2.4 & 0.07 \\
\hline Pluronic F108 & $7-45$ & 33.4 & 2.0 \\
\hline Pluronic F108-amine & - & 27.1 & 0.90 \\
\hline from [36, 42, 43] & \multicolumn{3}{|l}{} \\
\end{tabular}

tation wavelength was $320 \mathrm{~nm}$ with a monochromator slit width of $5 \mathrm{~nm}$ while emission slit was set to $2 \mathrm{~nm}$. Ratio of intensity of the first $\left(I_{1}\right.$ at $\left.373 \mathrm{~nm}\right)$ and third peaks $\left(I_{3}\right.$ at $\left.383 \mathrm{~nm}\right)$ is a sensitive parameter characterizing the polarity of the probe's environment. Strong emission at $383 \mathrm{~nm}$ with low $I_{1} / I_{3}$ value indicates low polarity of the environment. Hence $I_{1} / I_{3}$ is expected to decrease at the onset of the micelle formation, reflecting a preferential solubilization of pyrene into a less polar microenvironment $[37,38]$.

\subsection{Preparation of Pluronic stabilized PLGA NPs}

PLGA NPs were prepared in triplicates by the nanoprecipitation method similar to that employed previously [23, 39]. Briefly PLGA was dissolved in acetone at a concentration of $10 \mathrm{~g} / \mathrm{L} .6 \mathrm{~mL}$ of the organic solution was added to $24 \mathrm{~mL}$ aqueous solution of Pluronic or Pluronic-amine under magnetic stirring (500 rpm). Pluronic concentration in the aqueous phase was $2 \mathrm{~g} / \mathrm{L}$. NPs were formed and stirring was continued overnight to achieve the complete evaporation of acetone. The aqueous PLGA sol was centrifuged at $3500 \mathrm{~g}$ for $10 \mathrm{~min}$ to remove the possible polymer aggregates. The sol obtained as supernatant was further purified by centrifugation at $12000 \mathrm{~g}$ for 15 min where the supernatant was removed and the pellet containing the NPs was re-dispersed in doubly distilled water. This procedure was repeated three times in order to remove the dissolved Pluronic from the aqueous medium.

\subsubsection{Characterization of the size of NPs}

Average hydrodynamic size and polydispersity of the PLGA NPs were determined using a dynamic light scattering (DLS) system (Brookhaven Instruments, USA) consisting of a BI-200SM goniometer and a BI-9000AT digital autocorrelator. As a light source a Coherent Genesis MX488-1000STM laser-diode system operating at $488 \mathrm{~nm}$ wavelength and emitting vertically polarized light was used. Measurements were carried out at a detection angle of $90^{\circ}$ and a temperature of $25^{\circ} \mathrm{C}$ with nanoparticle sample appropriately diluted with doubly distilled water. The recorded autocorrelation functions were analyzed by the second order cumulant expansion method.

\subsubsection{Determination of zeta potential of NPs}

The measurement of electrophoretic mobility of nanoparticles was carried out by means of Malvern Zetasizer Nano $\mathrm{Z}$ apparatus at 25 and $37^{\circ} \mathrm{C}$. Smoluchowski approximation was used to calculate zeta potential from mobility values. Particles were surveyed in aqueous salt solution with constant ionic strength $(2 \mathrm{mM} \mathrm{NaCl})$.

\subsubsection{Characterization of colloidal stability of NPs}

The effect of ionic strength on the colloidal stability of PLGA nanoparticles was investigated by adding salt solutions with increasing concentration to the sols. Sodium chloride and calcium acetate solutions were used as coagulation media. Experiments were carried out at 25 and $37^{\circ} \mathrm{C}$. Turbidity was measured with a spectrophotometer at a wavelength of $400 \mathrm{~nm}$ after incubation time of $15 \mathrm{~min}$. The concentration of electrolyte which resulted in three times higher absorbance comparing to that of the original sol was used to estimate the critical aggregation concentrations (CAC). Higher CAC indicates the better colloidal stability of NP system. The electrolyte concentration was increased in $0.1 \mathrm{M}$ steps during the experiments. No deviation was found in CAC during measurements of three separated batches.

\section{Results and discussion \\ 3.1. Preparation and characterization of Pluronic-amines}

Amine end group derivatives of Pluronic F68, F127 and F108 were prepared via reductive amination of the partially oxidized hydroxyl end groups (Figure 1). Products were acquired with $60-80 \%$ yield following the two step reaction. The exact determination of the conversion of the end groups of Pluronics poses a significant challenge. Mass spectrometry could not be employed due to the similarity in the mass of the amine and hydroxyl groups. Classical analytical methods based on the determination of total nitrogen content were also not feasible because of the low 


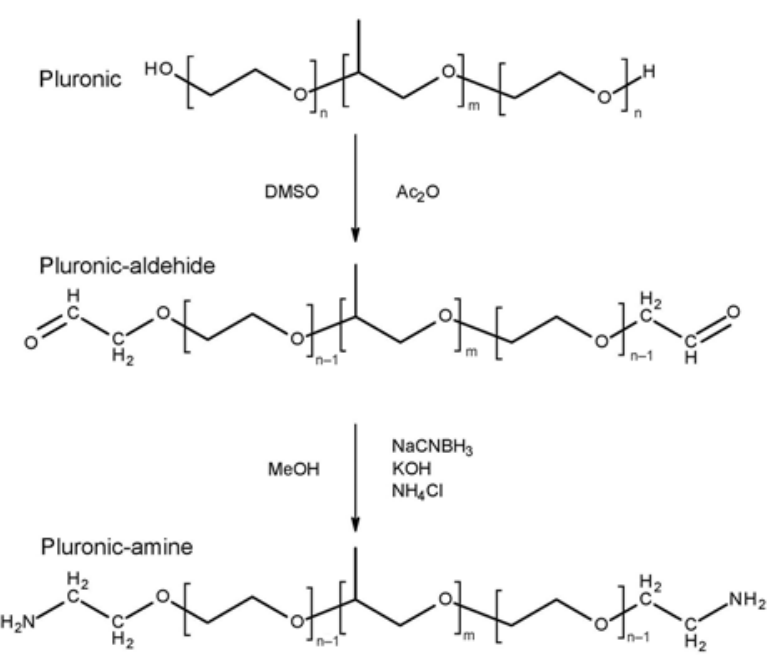

Figure 1. Two step conversion of Pluronics into Pluronicamines

nitrogen content (appr. $0.1 \mathrm{wt} \%$ ) of the polymeric compound.

Fluorescamine assay was employed as a selective and sensitive technique to obtain the amine content of the products. Fluorescamine, a nonfluorescent compound, reacts with primary amines to form pyrrolinones. Upon excitation at $390 \mathrm{~nm}$ these compounds exhibit strong fluorescence in the 450 to $550 \mathrm{~nm}$ range. Addition of fluorescamine to the products resulted in the formation of fluorescent species indicating the presence of primary amines. For the quantitative determination a calibration curve was recorded with hexylamine in the concentration range of 0 $60 \mu \mathrm{mol} / \mathrm{L}$ while the Pluronic samples were measured at $0.2 \mathrm{~g} / \mathrm{L}$ concentration (Figure 2). The fluorescent spectra of the fluorophores formed from the Pluronics and hexylamine had similar shape. Only a slight shift of around $3 \mathrm{~nm}$ was observed between the

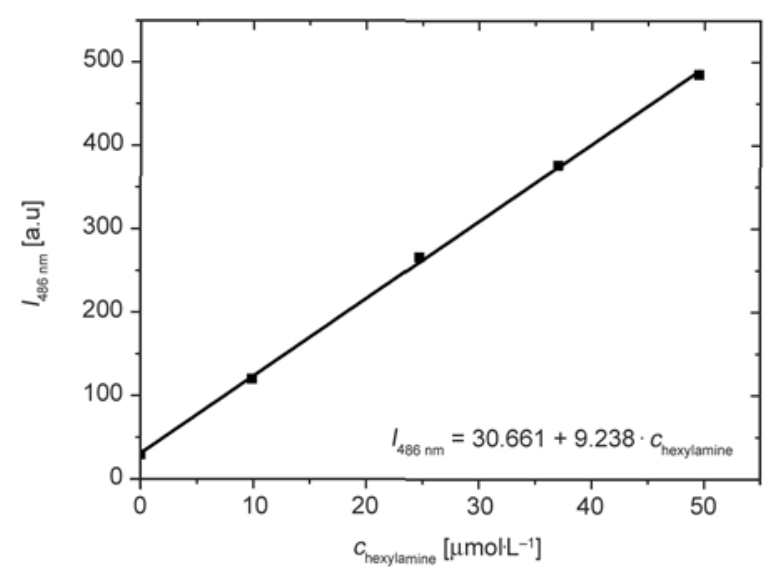

Figure 2. Fluorescence intensity calibration curve recorded with the hexylamine fluorophore

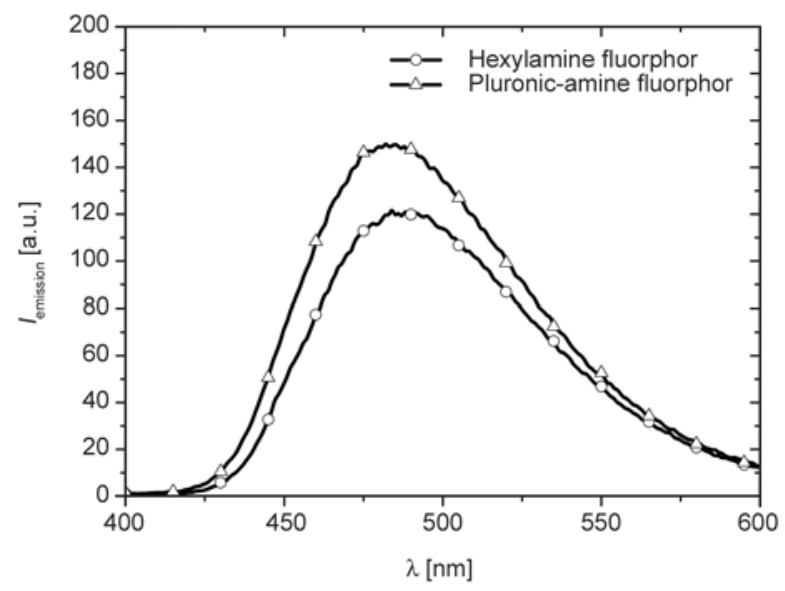

Figure 3. Fluorescent spectra of the hexylamine and Pluronic F127-amine fluorophores

intensity maxima of the two amine functional materials (Figure 3). The method proved to be robust enough for the determination of the amine content of the samples as measured intensities were within 5\% error range for the triplicate measurements.

Taking into consideration the average molar masses and structure of the Pluronics the obtained end group amine conversions were 38,41 and $22 \%$ for F68, F127 and F108 respectively with a standard deviation of $1-2 \%$. The batch to batch reproducibility of conversion was also within 5\% error. Since the chemical yield of the fluorophore is dependent to some extent on the structure of the amine [40] the acquired conversion values can be considered as approximates.

The transformation reaction to prepare amino-functional Pluronic was repeated in aqueous medium instead of methanol. Only 8 and $14 \%$ conversion was determined for F68 and F127 and practically none for F108. These findings indicate that the presence of water reduces the proceeding of the reaction.

The presence and reactivity of functional amino groups on Pluronic molecules were investigated by conjugation with Boc- or Fmoc-L-phenylalanine. The success of the conjugation reaction was checked by ninhydrine test and TLC. ${ }^{1} \mathrm{H}$ NMR spectra of the Boc-Phe and Fmoc-Phe-coupled Pluronic-amines clearly indicated the appearance of aromatic hydrogens in the range of 6.5-7.8 ppm. The ${ }^{1} \mathrm{H}$ NMR spectra of the Pluronic F127-amine and its Fmoc-Phe derivative is shown in Figure 4.

The strong singlet peak at $3.65 \mathrm{ppm}$ belongs to the $\mathrm{CH}_{2}$ groups of the PEO blocks. The two complex multiplets with 2:1 intensity ratio in the range of 3.3-3.6 ppm are the signals of $-\mathrm{CH}-\mathrm{CH}_{2}-$ protons of 


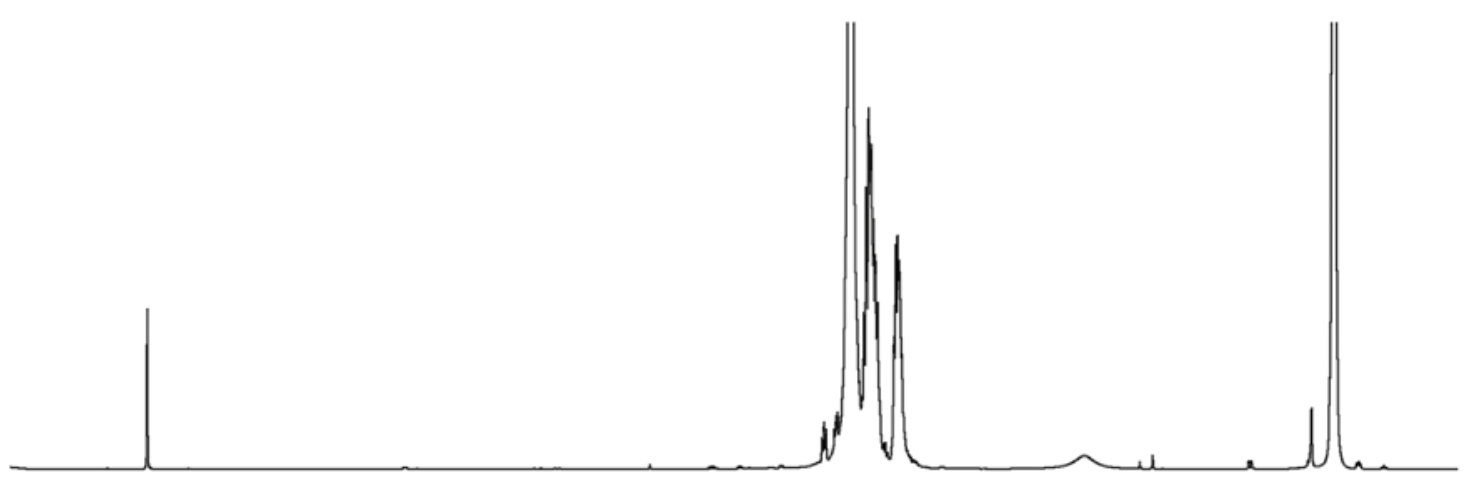

a)

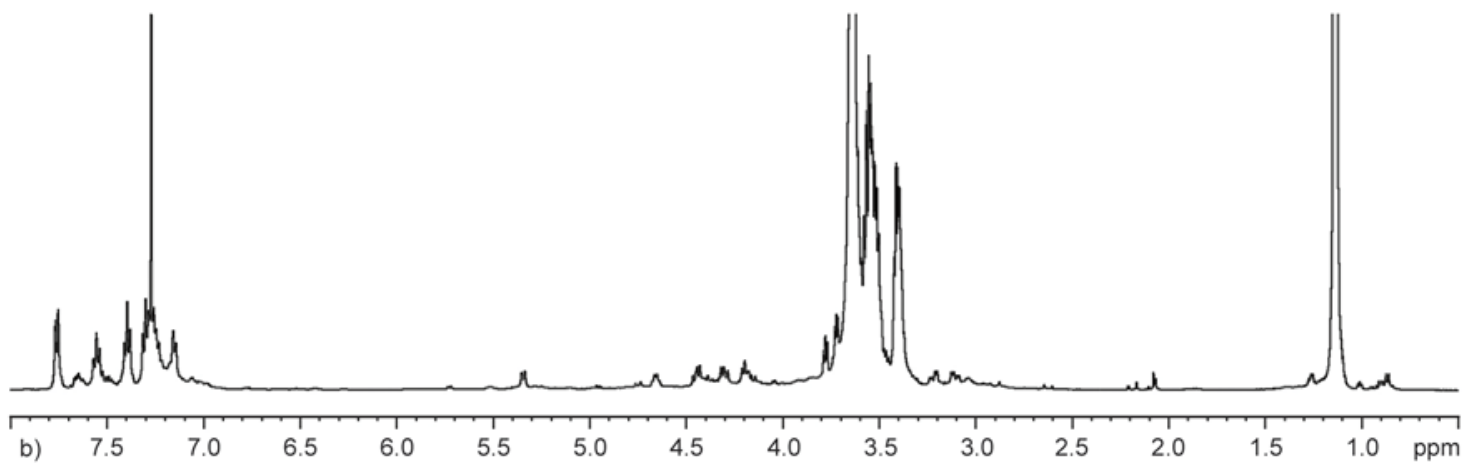

Figure 4. ${ }^{1} \mathrm{H}$ NMR spectra of the Pluronic F127-amine (a) and Pluronic F127-Phe-Fmoc (b)

the PPO block while the peak at 1.14 ppm belongs to PPO methyl groups. The amine derived Pluronic F127 exhibits a broad signal at $2.434 \mathrm{ppm}$, which indicates the presence of terminal amine groups.

Pluronic F127-Phe-Fmoc shows very similar signals to the amine derived F127 in the spectrum ranges given above. The major difference is the vanishing amine signal at $2.434 \mathrm{ppm}$ and the new aromatic protons in the range of $7-8 \mathrm{ppm}$. There are new weak proton signals in the 4.0-5.5 ppm and 2.5$3.3 \mathrm{ppm}$ regions which belong to the aliphatic protons of the terminal Phe-Fmoc groups.

The aromatic region contains the multiplets of the ABCD spin systems of the two terminal fluorenyl groups and a strongly overlapping signal of the $\mathrm{AA}^{\prime} \mathrm{BB}^{\prime} \mathrm{C}$ system of the phenyl groups. The well separated doublet at $7.763 \mathrm{ppm}$ belongs to the overlapping aromatic protons in positions 4 and 5 of the fluorenyl group.

As amphiphatic block copolymers Pluronics selfassociate in aqueous solution forming micellar type aggregates. The concentration range where this association becomes dominant is a characteristic parameter depending on the composition of the molecule and environmental conditions as well. The critical micelle concentration (CMC) of Pluronic F68, F127 and F108 and their amine derivatives were deter-

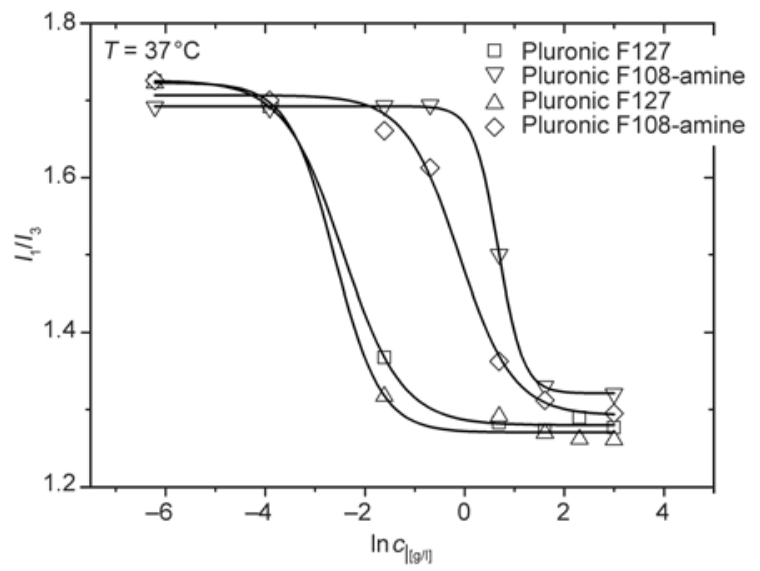

Figure 5. Pyrene $I_{1} / I_{3}$ values as a function of Pluronic concentration at $37^{\circ} \mathrm{C}$

mined in aqueous solution at two temperatures using the pyrene fluorescent probe method. Increasing the concentration of Pluronics a large decrease begins in the $I_{1} / I_{3}$ values after a given point indicating the appearance of hydrophobic domains due to the formation of micelles (Figure 5). At the end of this transition concentration range the solubilization of pyrene reaches the saturation which is represented by steady low value of $I_{1} / I_{3}$.

To resolve a definite concentration value of CMC the measured points were fitted with a decreasing Boltzmann type sigmoid, which is given by Equation (1): 
$y=\frac{A_{1}-A_{2}}{1+\mathrm{e}^{\left(\mathrm{x}-\mathrm{x}_{0}\right) / \Delta \mathrm{x}}}+A_{2}$

where the variable y corresponds to the $I_{1} / I_{3}$ values, while $x$ is the total Pluronic concentration. $A_{1}$ and $A_{2}$ are the upper and lower limiting values, $x_{0}$ is the centre of the sigmoid and $\Delta x$ is the width of the sigmoid. According to Aguiar et al. [41] the CMC should be given as $x_{0}$ if the ratio $x_{0} / \Delta_{\mathrm{x}}<10$ and as $\left(x_{0}+2 \Delta x\right)$ otherwise. Since the $x_{0} / \Delta_{\mathrm{x}}<10$ condition was valid in all of our cases $x_{0}$ was selected as CMC. The determined CMC values are shown in Table 1.

CMC values obtained for Pluronics F127 and F108 at $25^{\circ} \mathrm{C}$ are in accordance with the results of other studies being within the range reported previously [36, $42,43]$. That is valid for Pluronic F68 too, which did not show association in the investigated concentration range up to $100 \mathrm{~g} / \mathrm{L}$.

Considering the Pluronic-amines the CMC of the amine derivatives were found to be in the same concentration range as the corresponding starting material signifying that the present chemical modification of the end groups does not change the association behavior of the Pluronics. Comparing the shapes of $I_{1} / I_{3}$ vs concentration curves for the Pluronics and their amine derivatives however some differences can be observed (Figure 5). The transition of the pyrene probe from a hydrophilic to a hydrophobic environment occurs over a relatively wide concentration range especially for the amine derivatives which could be related to the dispersity of the polymer. This makes the determination of the CMC defined as the centre of the fitted sigmoid somewhat ambiguous. That might be the explanation for the shift of CMC values given in Table 1.

As it is characteristic for PEO-containing surfactants in general a decrease in the micellization concentration was observed at higher temperature. The decrease of $\mathrm{CMC}$ is about one order of magnitude in the $25-37^{\circ} \mathrm{C}$ temperature range studied here.

\subsection{Preparation and characterization Pluronic stabilized nanoparticles}

Pluronic and Pluronic-amine stabilized PLGA nanoparticles were prepared by nanoprecipitation. The NPs obtained applying Pluronic F127 and Pluronic F108 as well as their amine derivatives were characterized by dynamic light scattering and zeta potential measurements. The average hydrodynamic
Table 2. Average hydrodynamic diameter $(d)$, polydispersity (PD) and zeta potential ( $\zeta$ ) of PLGA NPs ${ }^{*}$

\begin{tabular}{|c|c|c|c|c|}
\hline \multirow[t]{2}{*}{ Stabilizer } & \multirow{2}{*}{$\begin{array}{c}d \\
{[\mathrm{~nm}]}\end{array}$} & \multirow[t]{2}{*}{ PD } & \multicolumn{2}{|c|}{$\begin{array}{c}\zeta \\
{[\mathrm{mV}]}\end{array}$} \\
\hline & & & $25^{\circ} \mathrm{C}$ & $37^{\circ} \mathrm{C}$ \\
\hline Pluronic F127 & 114 & 0.05 & -11.3 & -11.9 \\
\hline Pluronic F127-amine & 116 & 0.08 & -6.5 & -5.7 \\
\hline Pluronic F108 & 115 & 0.06 & -14.2 & -13.4 \\
\hline Pluronic F108-amine & 118 & 0.06 & -10.2 & -10.9 \\
\hline
\end{tabular}

${ }^{*}$ Data were reproducible within $5 \%$ error between batches

diameter $(d)$, polydispersity $(P D)$ and zeta potential $(\zeta)$ of the particles are collected in Table 2.

Mean diameter of the particles was around $115 \mathrm{~nm}$ regardless of the stabilizer used. All samples showed a narrow size distribution with $P D$ values below 0.1 . The particles had a negative zeta potential due to the carboxylic end groups of the PLGA chains located on the particle surface and partly covered by Pluronic layer. The usage of Pluronic-amine decreased the potential which was valid for both Pluronics and at both temperatures investigated. That decrease in zeta potential is clearly due to the presence of protonated amine groups of Pluronic-amine.

Bare PLGA particles are stabilized in aqueous solution by electrostatic interactions due to their negative surface charge [44]. The stability of such systems vanishes above a certain electrolyte concentration due to the screening effect of ionic strength. Aggregation in these systems happens at as low $\mathrm{NaCl}$ concentration as $0.03 \mathrm{M}$ [23]. The role of Pluronic stabilizer however, is to give steric stability of NPs, making the colloidal system less sensitive to the variation of electrolyte concentration in the medium.

Colloidal stability of the Pluronic stabilized PLGA NPs was tested by measuring the turbidity of sols at different electrolyte concentrations in their aqueous media. The determined CAC values are listed in Table 3.

Table 3. Critical aggregation concentration (CAC) of PLGA NP systems stabilized by Pluronics and Pluronicamines checked by $\mathrm{NaCl}$ and $\mathrm{Ca}$-acetate at 25 and $37^{\circ} \mathrm{C}$

\begin{tabular}{|c|c|c|c|c|}
\hline \multirow{3}{*}{ Stabilizer } & \multicolumn{2}{|c|}{$\begin{array}{c}\mathbf{C A C} \\
{[\mathbf{M}]}\end{array}$} & \multicolumn{2}{|c|}{$\begin{array}{c}\text { CAC } \\
{[\mathbf{M}]}\end{array}$} \\
\hline & \multicolumn{2}{|c|}{$\mathrm{NaCl}$ electrolyte } & \multicolumn{2}{|c|}{ Ca-acetate electrolyte } \\
\hline & $25^{\circ} \mathrm{C}$ & $37^{\circ} \mathrm{C}$ & $25^{\circ} \mathrm{C}$ & $37^{\circ} \mathrm{C}$ \\
\hline Pluronic F127 & $>2.5$ & $>2.5$ & $>1$ & 0.9 \\
\hline Pluronic F127-amine & $>2.5$ & $>2.5$ & $>1$ & 1 \\
\hline Pluronic F108 & 2.0 & 1.8 & 0.6 & 0.6 \\
\hline Pluronic F108-amine & 2.3 & 1.8 & 0.6 & 0.6 \\
\hline
\end{tabular}


All samples showed a highly increased colloidal stability compared to the pure PLGA NP system. This is due to the steric stabilization by the Pluronic adsorption layer on the NP surface. Particles stabilized by Pluronic F127 and its amine derivative retained their stability at all concentrations that were tested, with $\mathrm{NaCl}$ up till $2.5 \mathrm{M}$ regardless of temperature. The particles stabilized with Pluronic F108 and F108-amine showed a slightly reduced but still remarkably high $\mathrm{CAC}$ values. These systems aggregated only around $2 \mathrm{M} \mathrm{NaCl}$. Destabilization occurred earlier at $37^{\circ} \mathrm{C}$ due to the fact that the hydrophobic interactions become more prominent between PEO chains with increasing temperature leading to a decrease in the hydration of the Pluronic adsorption layer $[45,46]$. Ca-acetate was also used as electrolyte to test the effect of divalent ions on the colloidal stability of the NPs. Due to its lower solubility the concentrations up till $1.0 \mathrm{M}$ were tested. NP systems stabilized by Pluronic F127 were stable, but aggregation occurred in systems where Pluronic F108 was applied. The slightly less stability obtained for all NP systems with Pluronic F108 or Pluronic F108amine comparing to the corresponding Pluronic F127 samples is probably due to the difference in chemical composition, the block ratio and hence in the structure of adsorption layers. It is important to note, however that practically no difference was observed in the stabilization potential of Pluronics and their amine derivatives, both forms were effective.

\section{Conclusions}

End group derivative of various Pluronics were synthesized in a simple chemical procedure to obtain Pluronic-amine. The formation of the product was confirmed by the fluorescamine method and NMR analysis of the Boc-Phe-OH and Fmoc-Phe-OH coupled derivatives.

As verified by the CMC measurements the chemical modification did not alter significantly the polarity and association property of the molecules in aqueous environment.

The Pluronic-amines were applied as steric stabilizers for PLGA NPs prepared by the nanoprecipitation technique. The particles formed were in the 110 $120 \mathrm{~nm}$ range making them ideal for drug delivery applications. No significant changes were observed in the size of the particles when the original Pluronics and their amine derivatives were applied. The presence of the positively charged end groups were however detectable in electrophoretic mobility measurements by a shift in the zeta potential. The colloidal stability of the PLGA NPs was sufficiently high at 25 and $37^{\circ} \mathrm{C}$ making them suitable for use in biological environments with high ionic strengths.

The possibility for further chemical modifications of the Pluronic-amines was also demonstrated through the chemical binding of Boc-Phe-OH and FmocPhe-OH to the amine terminal. Such type of modifications open the way for convenient conjugation with e.g. host cell specific peptides as selective targeting moieties making achievable efficient drug delivery.

Direct experimental data support the conclusion that the membrane affinity of Pluronic derivatives (i.e. Pluronic-polyamine) was improved when compared to that of Pluronic [27, 47]. Based on these data we may assume that this feature will be present when Pluronic-amines are on the surface of PLGA NPs. Therefore we may expect that NPs containing Pluronic-amine surface layer show higher cellular uptake rate. This assumption was supported by our experimental data on drug loaded PLGA NPs [48]. When the carriers were functionalized by Pluronic F127 and Pluronic-F127-amine highly increased in vitro efficiency was achieved for an antituberculotic drug candidate. The reason was shown to be the ability for the particles to enter the host cells of the bacteria. When the Pluronic-amine derivative was used for the stabilization of the particles increased internalization and intracellular antibacterial effect was obtained. The presence of cationic charges on the particle surface was shown to increase the non-specific cell membrane affinity of the particles leading to increased cellular uptake rate [48].

\section{Acknowledgements}

This work was supported by the National Science Foundation: OTKA 104928, 104275, OTKA NK 105898 and TÁMOP 4.2.4. A/2-11-1-2012-0001 'National Excellence Program'.

\section{References}

[1] Ganguly K., Chaturvedi K., More U. A., Nadagouda M. N., Aminabhavi T. M.: Polysaccharide-based micro/ nanohydrogels for delivering macromolecular therapeutics. Journal of Controlled Release, 193, 162-173 (2014).

DOI: $10.1016 /$ j.jconrel.2014.05.014 
[2] Mohanraj V. J., Chen Y.: Nanoparticles - A review. Tropical Journal of Pharmaceutical Research, 5, 561-573 (2006).

DOI: $10.4314 /$ tjpr.v5i1.14634

[3] Soppimath K. S., Aminabhavi T. M., Kulkarni A. R., Rudzinski W. E.: Biodegradable polymeric nanoparticles as drug delivery devices. Journal of Controlled Release, 70, 1-20 (2001).

DOI: 10.1016/S0168-3659(00)00339-4

[4] Mu L., Seow P. H.: Application of TPGS in polymeric nanoparticulate drug delivery system. Colloids and Surfaces B: Biointerfaces, 47, 90-97 (2006).

DOI: 10.1016/j.colsurfb.2005.08.016

[5] Karger-Kocsis J., Kéki S.: Biodegradable polyesterbased shape memory polymers: Concepts of (supra) molecular architecturing. Express Polymer Letters, 8, 397-412 (2014).

DOI: $10.3144 /$ expresspolymlett.2014.44

[6] Mundargi R. C., Babu V. R., Rangaswamy V., Patel P., Aminabhavi T. M.: Nano/micro technologies for delivering macromolecular therapeutics using poly $(\mathrm{D}, \mathrm{L}-$ lactide-co-glycolide) and its derivatives. Journal of Controlled Release, 125, 193-209 (2008).

DOI: $10.1016 /$ j.jconrel.2007.09.013

[7] Fessi H., Puisieux F., Devissaguet J. P., Ammoury N., Benita S.: Nanocapsule formation by interfacial polymer deposition following solvent displacement. International Journal of Pharmaceutics, 55, R1-R4 (1989). DOI: 10.1016/0378-5173(89)90281-0

[8] Pitt C. G., Gratzl M. M., Kimmel G. L., Surles J., Schindler A.: Aliphatic polyesters II. The degradation of poly (DL-lactide), poly (epsilon-caprolactone), and their copolymers in vivo. Biomaterials, 2, 215-220 (1981).

[9] Li S. M., Garreau H., Vert M.: Structure-property relationships in the case of the degradation of massive poly( $\alpha$-hydroxy acids) in aqueous media, Part 3 Influence of the morphology of poly(L-lactic acid). Journal of Materials Science: Materials in Medicine, 1, 198206 (1990).

DOI: $10.1007 / \mathrm{BF} 00701077$

[10] Pistner H., Bendi D. R., Mühling J., Reuther J. F.: Poly (L-lactide): A long-term degradation study in vivo: Part III. Analytical characterization. Biomaterials, 14, 291298 (1993).

DOI: 10.1016/0142-9612(93)90121-H

[11] Czifrák K., Karger-Kocsis J., Daróczi L., Zsuga M., Kéki S.: Poly( $\varepsilon$-caprolactone) and Pluronic diol-containing segmented polyurethanes for shape memory performance. Macromolecular Chemistry and Physics, 215, 1896-1907 (2014).

DOI: $10.1002 /$ macp. 201400237

[12] Storm G., Belliot S. O., Daemen T., Lasic D. D.: Surface modification of nanoparticles to oppose uptake by the mononuclear phagocyte system. Advanced Drug Delivery Reviews, 17, 31-48 (1995).

DOI: 10.1016/0169-409X(95)00039-A
[13] Moghimi S. M.: Re-establishing the long circulatory behaviour of poloxamine-coated particles after repeated intravenous administration: Applications in cancer drug delivery and imaging. Biochimica et Biophysica Acta - General Subjects, 1472, 399-403 (1999). DOI: $10.1016 / \mathrm{S} 0304-4165(99) 00157-9$

[14] Gyulai G., Pénzes Cs. B., Mohai M., Lohner T., Petrik P., Kurunczi S., Kiss É.: Interfacial properties of hydrophilized poly(lactic-co-glycolic acid) layers with various thicknesses. Journal of Colloid and Interface Science, 362, 600-606 (2011).

DOI: $10.1016 /$ j.jcis.2011.06.055

[15] Kiss É., Gölander C-G.: Static and dynamic wetting behavior of poly(ethylene oxide) layer formed on mica substrate. Colloids and Surfaces, 58, 263-270 (1991). DOI: 10.1016/0166-6622(91)80226-E

[16] Kiss É., Dravetzky K., Hill K., Kutnyánszky E., Varga A.: Protein interaction with a Pluronic-modified poly (lactic acid) Langmuir monolayer. Journal of Colloid and Interface Science, 325, 337-345 (2008).

DOI: $10.1016 /$ j.jcis.2008.05.057

[17] Kiss É., Kutnyánszky E., Bertóti I.: Modification of poly(lactic/glycolic acid) surface by chemical attachment of poly(ethylene glycol). Langmuir, 26, 14401444 (2010).

DOI: $10.1021 / 1 \mathrm{a} 903373 \mathrm{~g}$

[18] Cheng J., Teply B. A., Sherifi I., Sung J., Luther G., Gu F. X., Levy-Nissenbaum E., Radovic-Moreno A. F., Langer R., Farokhzad O. C.: Formulation of functionalized PLGA-PEG nanoparticles for in vivo targeted drug delivery. Biomaterials, 28, 869-876 (2007). DOI: 10.1016/j.biomaterials.2006.09.047

[19] Moghimi S. M.: Prolonging the circulation time and modifying the body distribution of intravenously injected polystyrene nanospheres by prior intravenous administration of poloxamine-908. A 'hepatic-blockade' event or manipulation of nanosphere surface in vivo? Biochimica et Biophysica Acta - General Subjects, 1336, 1-6 (1997). DOI: $10.1016 / \mathrm{S} 0304-4165(97) 00060-3$

[20] Redhead H. M., Davis S. S., Illum L.: Drug delivery in poly(lactide-co-glycolide) nanoparticles surface modified with poloxamer 407 and poloxamine 908: In vitro characterisation and in vivo evaluation. Journal of Controlled Release, 70, 353-363 (2001). DOI: 10.1016/S0168-3659(00)00367-9

[21] Kiss É., Varga A., Vargha-Butler É. I.: Interfacial behaviour of poly(lactic acid) and Pluronic6400 mixed monolayers at the air-water interface. Physical Chemistry Chemical Physics, 6, 1575-1579 (2004). DOI: $10.1039 / \mathrm{B} 312950 \mathrm{~K}$

[22] Kiss É., Takács M. G., Bertóti I., Vargha-Butler E. I.: Surface properties of poly(lactic/glycolic acid)Pluronic ${ }^{\circledR}$ blend films. Polymers for Advanced Technologies, 14, 839-846 (2003).

DOI: $10.1002 /$ pat.404 
[23] Gyulai G., Pénzes Cs. B., Mohai M., Csempesz F., Kiss É.: Influence of surface properties of polymeric nanoparticles on their membrane affinity. European Polymer Journal, 49, 2495-2503 (2013).

DOI: $10.1016 /$ j.eurpolymj.2013.02.024

[24] Neal J. C., Stolnik S., Garnett M. C., Davis S. S., Illum L.: Modification of the copolymers Poloxamer 407 and Poloxamine 908 can affect the physical and biological properties of surface modified nanospheres. Pharmaceutical Research, 15, 318-324 (1998).

DOI: 10.1023/A:1011987206722

[25] Yaniç C., Bredenkamp M. W., Jacobs E. P., Spies H. S. C., Swart P.: NMR spectroscopy as basis for characterization of Pluronic ${ }^{\mathbb{R}}$ F108 and its derivatives. Journal of Applied Polymer Science, 78, 109-117 (2000).

DOI: $10.1002 / 1097-4628(20001003) 78: 1<109::$ AIDAPP140>3.0.CO;2-6

[26] Vinogradov S. V., Bronich T. K., Kabanov A. V.: Selfassembly of polyamine-poly(ethylene glycol) copolymers with phosphorothioate oligonucleotides. Bioconjugate Chemistry, 9, 805-812 (1998).

DOI: $10.1021 /$ bc $980048 \mathrm{q}$

[27] Yi X., Batrakova E., Banks W. A., Vinogradov S., Kabanov A. V.: Protein conjugation with amphiphilic block copolymers for enhanced cellular delivery. Bioconjugate Chemistry, 19, 1071-1077 (2008).

DOI: $10.1021 /$ bc $700443 \mathrm{k}$

[28] Zalipsky S.: Functionalized poly(ethylene glycols) for preparation of biologically relevant conjugates. Bioconjugate Chemistry, 6, 150-165 (1995).

DOI: $10.1021 / \mathrm{bc} 00032 \mathrm{a} 002$

[29] Harris J. M., Struck E. C., Case M. G., Paley M. S., Yalpani M., Van Alstine J. M., Brooks D. E.: Synthesis and characterization of poly(ethylene glycol) derivatives. Journal of Polymer Science: Polymer Chemistry Edition, 22, 341-352 (1984).

DOI: $10.1002 /$ pol.1984.170220207

[30] Tatur S., Maccarini M., Barker R., Nelson A., Fragneto G.: Effect of functionalized gold nanoparticles on floating lipid bilayers. Langmuir, 29, 6606-6614 (2013). DOI: $10.1021 / 1 \mathrm{a} 401074 \mathrm{y}$

[31] Kiss É., Bertóti I.: Preparation and characterization of PEO grafted surfaces by wettability measurements. Progress in Colloid and Polymer Science, 97, 21-26 (1994).

DOI: $10.1007 / \mathrm{BFb} 0115129$

[32] Lillie R. D.: Simplification of the manufacture of schiff reagent for use in histochemical procedures. Biotechnic and Histochemistry, 26, 163-165 (1951).

DOI: $\underline{10.3109 / 10520295109113200}$

[33] Böhlen P., Stein S., Dairman W., Udenfriend S.: Fluorometric assay of proteins in the nanogram range. Archives of Biochemistry and Biophysics, 155, 213-220 (1973). DOI: 10.1016/S0003-9861(73)80023-2
[34] Belal F., Abdine H., Al-Majed A., Khalil N. Y.: Spectrofluorimetric determination of vigabatrin and gabapentin in urine and dosage forms through derivatization with fluorescamine. Journal of Pharmaceutical and Biomedical Analysis, 27, 253-260 (2002). DOI: $10.1016 / \mathrm{S} 0731-7085(01) 00503-9$

[35] Adamou R., Coly A., Douabalé S. E., Saleck M. L. O. C. O., Gaye-Seye M. D., Tine A.: Fluorimetric determination of histamine in fish using micellar media and fluorescamine as labelling reagent. Journal of Fluorescence, 15, 679-688 (2005).

DOI: $10.1007 / \mathrm{s} 10895-005-2975-7$

[36] Alexandridis P., Holzwarth J. F., Hatton T. A.: Micellization of poly(ethylene oxide)-poly(propylene oxide)poly(ethylene oxide) triblock copolymers in aqueous solutions: Thermodynamics of copolymer association. Macromolecules, 27, 2414-2425 (1994).

DOI: $10.1021 / \mathrm{ma} 00087 \mathrm{a} 009$

[37] Goddard E. D., Turro N. J., Kuo P. L., Ananthapadmanabhan K. P.: Fluorescence probes for critical micelle concentration determination. Langmuir, 1, 352-355 (1985).

DOI: $10.1021 / \mathrm{la} 00063 \mathrm{a} 015$

[38] Wang Y., Zhang M., Moers C., Chen S., Xu H., Wang Z., Zhang X., Li Z.: Block copolymer aggregates with photo-responsive switches: Towards a controllable supramolecular container. Polymer, 50, 4821-4828 (2009).

DOI: $10.1016 /$ j.polymer.2009.08.005

[39] Kiss É., Schnöller D., Pribranská K., Hill K., Pénzes Cs. B., Horváti K., Bösze S.: Nanoencapsulation of antitubercular drug isoniazid and its lipopeptide conjugate. Journal of Dispersion Science and Technology, 32, 1728-1734 (2011). DOI: 10.1080/01932691.2011.616128

[40] de Bernardo S., Weigele M., Toome V., Manhart K., Leimgruber W. B. P., Böhlen P., Stein S., Udenfriend S.: Studies on the reaction of fluorescamine with primary amines. Archives of Biochemistry and Biophysics, 163, 390-399 (1974).

DOI: $10.1016 / 0003-9861(74) 90490-1$

[41] Aguiar J., Carpena P., Molina-Bolívar J. A., Carnero Ruiz C.: On the determination of the critical micelle concentration by the pyrene 1:3 ratio method. Journal of Colloid and Interface Science, 258, 116-122 (2003). DOI: 10.1016/S0021-9797(02)00082-6

[42] Chu B., Zhou Z.: Physical chemistry of polyoxyalkylene block copolymer surfactants. in 'Nonionic surfactants: Polyoxyalkylene block copolymers' (ed.: Nace V. M.) Marcel Dekker, New York 78-80 (1996).

[43] Lopes J. R., Loh W.: Investigation of self-assembly and micelle polarity for a wide range of ethylene oxidepropylene oxide-ethylene oxide block copolymers in water. Langmuir, 14, 750-756 (1998).

DOI: $10.1021 / 1 \mathrm{a} 9709655$ 
[44] Santander-Ortega M. J., Jódar-Reyes A. B., Csaba N., Bastos-González D., Ortega-Vinuesa J. L.: Colloidal stability of Pluronic F68-coated PLGA nanoparticles: A variety of stabilisation mechanisms. Journal of Colloid and Interface Science, 302, 522-529 (2006). DOI: $10.1016 /$ j.jcis.2006.07.031

[45] Claesson P. M., Kjellander R., Stenius P., Christenson H. K.: Direct measurement of temperature-dependent interactions between non-ionic surfactant layers. Journal of the Chemical Society, Faraday Transactions 1: Physical Chemistry in Condensed Phases, 82, 27352746 (1986).

DOI: $10.1039 / \mathrm{F} 19868202735$

[46] Kiss É.: Temperature dependence of bovine serum albumin adsorption onto a poly(ethylene oxide)-grafted surface. Colloids and Surfaces A: Physicochemical and Engineering Aspects, 76, 135-140 (1993). DOI: 10.1016/0927-7757(93)80071-L
[47] Fan W., Wu X., Ding B., Gao J., Cai Z., Zhang W., Yin D., Wang X., Zhu Q., Liu J., Ding X., Gao S.: Degradable gene delivery systems based on Pluronics-modified low-molecular-weight polyethylenimine: Preparation, characterization, intracellular trafficking, and cellular distribution. International Journal of Nanomedicine, 7, 1127-1138 (2012). DOI: $10.2147 /$ IJN.S27117

[48] Kiss É., Gyulai G., Pénzes Cs. B., Idei M., Horváti K., Bacsa B., Bősze S.: Tuneable surface modification of PLGA nanoparticles carrying new antitubercular drug candidate. Colloids and Surfaces A: Physicochemical and Engineering Aspects, 458, 178-186 (2014). DOI: $10.1016 /$ j.colsurfa.2014.05.048 